\title{
Magnetic Catalysis in the Higher-Order Quark Sigma Model
}

\author{
M. Abu-Shady \\ Department of Applied Mathematics, Faculty of Science, Menoufia University, Egypt \\ Email: dr.abushady@gmail.com
}

\begin{abstract}
The effect of the higher-order mesonic interactions in the presence of an external magnetic field is investigated within the framework of the chiral symmetry breaking mechanism. The effect of higher-order mesonic potential is employed and numerically solved in the mean-field approximation. The chiral symmetry breaking increases with ascending magnetic field. Two sets of free parameterization are investigated on the magnetic catalysis. A comparison is discussed with the other studies and Lattice QCD. The obtained results are included that the higher-order quark sigma model gives good description for the magnetic catalysis.
\end{abstract}

Keywords: Chiral lagrangian density, magnetic catalysis, chiral symmetry breaking.

\section{Introduction}

Chiral symmetry breaking is an important phenomenon in hadron physics and has a fundamental importance for hadron properties. The difficulties involved in obtaining low-energy properties directly from QCD, the fundamental theory of strong interactions, have motivated the construction of effective models due to their simplicity and effectiveness in describing hadrons at low energies [1]. The linear sigma model was first proposed in the 1960s as a model for pion-nucleon interactions and later has been offered for strong nuclear interactions [2]. The model was first proposed in the 1960s as a model for pion-nucleon interactions. Today it serves as an effective model for the low-energy phase (zero temperature) of quantum chromodynamics $[3,4,5,6]$ and its modification is suggested as in works $[7,8,9,10,11]$ to provide a good description of the baryon properties. In addition, the quark sigma model is successfully applied to the description of static and dynamic baryon properties at finite temperature and density as in Refs. $[12,13,14,15,16,17]$.

The study of the influence of external magnetic fields on the fundamental properties of quantum chromodynamic (QCD) theory, confinement and dynamical chiral symmetry breaking is still a matter of great interest both in theoretical and experimental activities $[18,19,20,21]$. The chiral symmetry structure in the original sigma model within the presence of an external uniform magnetic field is investigated [22]. Also in another work [23] the chiral phase transition in the presence of electromagnetic field was examined and the team of that work found that the magnetic field enhances the chiral symmetry breaking in the NJL model. Later, the quark condensate was studied in the presence of external magnetic field using the Schwinger-Dyson equation[24,25]. To study the influence of magnetic field the proper time method was applied to a four-fermion interaction model and noted that an external magnetic field has the effect of enhancing chiral symmetry breaking $[26,27,28]$.

The higher-order mesonic interactions play an important role to test nonperturbative chiral dynamics [29]. It is useful to specify that the hadron properties are improved in comparison with other models and in a good agreement with experimental data by including higher-order mesonic interactions as in $[11,30]$.

In this paper, we investigate the effect of higher-order mesonic interactions on the chiral symmetry breaking in the presence of an external magnetic field within the framework of quark sigma model. In addition, a new parametrization for sigma mass and coupling constant on the behavior of the phase transition is studied. So far no attempt has been made to include higher-order mesonic interactions on the chiral symmetry breaking as far as an external magnetic field exists.

This paper is organized as follows: The original sigma model is briefly presented in Sec. 2. Next, the effective higher-order mesonic potential in the presence of external magnetic field is presented in Sec. 3. The results are discussed and compared with the other models in Secs. 4 and 5, respectively. Finally, the summary and conclusion part comes in Sec. 6 . 


\section{The Chiral Sigma Model with Original Effective Potential}

The interactions of quarks via the exchange of $\sigma$-and $\pi$-meson fields are given by the Lagrangian density [3] as follows:

$$
L(r)=i \bar{\Psi} \gamma^{\mu} \partial_{\mu} \Psi+\frac{1}{2}\left(\partial_{\mu} \sigma \partial^{\mu} \sigma+\partial_{\mu} \pi . \partial^{\mu} \pi\right)+g \bar{\Psi}\left(\sigma+i \gamma_{5} \tau . \pi\right) \Psi-U_{1}(\sigma, \pi),
$$

with

$$
U_{1}(\sigma, \pi)=\frac{\lambda^{2}}{4}\left(\sigma^{2}+\pi^{2}-\nu^{2}\right)^{2}+m_{\pi}^{2} f_{\pi} \sigma
$$

where $U_{1}(\sigma, \pi)$ is the meson-meson interaction potential. $\Psi, \sigma$ and $\pi$ are the quark, sigma, and pion fields, respectively. In the mean-field approximation, the meson fields are treated as time-independent classical fields. This means that we replace the power and products of the meson fields by corresponding powers and the products of their expectation values. The meson-meson interactions in Eq. (2) lead to hidden chiral $S U(2) \times S U(2)$ symmetry with $\sigma(r)$ taking on a vacuum expectation value

$$
\langle\sigma\rangle=-f_{\pi},
$$

where $f_{\pi}=93 \mathrm{MeV}$ is the pion decay constant. The final term in Eq. (2) is included to break the chiral symmetry explicitly. It leads to the partial conservation of axial-vector isospin current (PCAC). The parameters $\lambda^{2}$ and $\nu^{2}$ can be expressed in terms of $f_{\pi}$, sigma and pion masses as,

$$
\begin{aligned}
& \lambda^{2}=\frac{m_{\sigma}^{2}-m_{\pi}^{2}}{2 f_{\pi}^{2}}, \\
& \nu^{2}=f_{\pi}^{2}-\frac{m_{\pi}^{2}}{\lambda^{2}} .
\end{aligned}
$$

\section{The Effective Higher-order Mesonic Potential in the Presence of Magnetic Field}

In this section, the higher-order mesonic potential $U_{2}(\sigma, \pi)$ is employed. In Eq. (6), the effective higherorder mesonic potential is included with the external magnetic field at zero temperature and density as follows,

$$
U_{\text {eff }}(\sigma, \pi)=U_{2}(\sigma, \pi)+U_{\text {Vacuum }}+U_{\text {Matter }}
$$

where

$$
\begin{aligned}
U_{2}(\sigma, \pi) & =\frac{\lambda_{1}^{2}}{4}\left(\sigma^{2}+\pi^{2}-\nu_{1}^{2}\right)^{2}+\frac{\lambda_{2}^{2}}{4}\left(\left(\sigma^{2}+\pi^{2}\right)^{2}-\nu_{2}^{2}\right)^{2} \\
& +m_{\pi}^{2} f_{\pi} \sigma
\end{aligned}
$$

In Eq. 7, the higher-order mesonic potential satisfies the chiral symmetry when $m_{\pi} \longrightarrow 0$ as well as in the standard potential in Eq. 2. Spontaneous chiral symmetry breaking gives a nonzero vacuum expectation value for $\sigma$ and the explicit chiral symmetry breaking term in Eq. 7 gives the pion mass.

$$
\langle\sigma\rangle=-f_{\pi}
$$

where

$$
\begin{gathered}
\lambda_{1}^{2}=\frac{1}{4 f_{\pi}^{2}}\left(m_{\sigma}^{2}-m_{\pi}^{2}\right), \\
\nu_{1}^{2}=f_{\pi}^{2}-\frac{m_{\pi}^{2}}{2 \lambda_{1}^{2}}, \\
\lambda_{2}^{2}=\frac{1}{16 f_{\pi}^{6}}\left(m_{\sigma}^{2}-3 m_{\pi}^{2}\right),
\end{gathered}
$$




$$
\nu_{2}^{2}=f_{\pi}^{4}-\frac{m_{\pi}^{2}}{4 \lambda_{2}^{2} f_{\pi}^{2}} .
$$

For details, see Refs. [11,30]. To include the external magnetic field in the present model, we follow Ref. [21]. So, the vacuum energy potential is given by

$$
U_{\text {Vacuum }}=\frac{N_{c} N_{f} g^{4}}{(2 \pi)^{2}}\left(\sigma^{2}+\pi^{2}\right)^{2}\left(\frac{3}{2}-\ln \left(\frac{g^{2}\left(\sigma^{2}+\pi^{2}\right)}{\Lambda^{2}}\right)\right),
$$

where $N_{c}=3$ and $N_{f}=2$ are color and flavor degrees of freedom. respectively, and $\Lambda$ is mass scale,

$$
U_{\text {Matter }}=\frac{N_{c}}{2 \pi^{2}} \sum_{f=u}^{d}\left(\left|q_{f}\right| B\right)^{2}\left[\zeta^{(1,0)}\left(-1, x_{f}\right)-\frac{1}{2}\left(x_{f}^{2}-x_{f}\right) \ln x_{f}+\frac{x_{f}^{2}}{4}\right]
$$

In Eq. 14, we have used $x_{f}=\frac{g^{2}\left(\sigma^{2}+\pi^{2}\right)}{\left(2\left|q_{f}\right| B\right)}$ and $\zeta^{(1,0)}\left(-1, x_{f}\right)=\left.\frac{d \zeta\left(z, x_{f}\right)}{d z}\right|_{z=-1}$ that represents the RiemannHurwitz function for detail, see Ref. [31], and also $\left|q_{f}\right|$ is the absolute value of quark electric charge in external magnetic field with intense $B$. In Eq. 6, the effect of the finite temperature and chemical potential is not included in the present model, in which the present model focus on the study of magnetic catalysis at low energy.

\section{Discussion of Results}

In this section, we study the effect of external magnetic field on the symmetry breaking in the presence of higher-order mesonic interactions and also discuss the effect of coupling constant $(g)$ and sigma mass $\left(m_{\sigma}\right)$ on symmetry breaking. For this purpose, we numerically calculate the effective potential in Eq. (6). The parameters of the present model are the coupling constant $g$ and the sigma mass $m_{\sigma}$. The choice of free parameters of $g$ and $m_{\sigma}$ is based on Ref. [4]. The parameters are usually chosen so that the chiral symmetry is spontaneously broken in the vacuum and the expectation values of the meson fields. In this work, we consider two different sets of parameters in order to get both high and low values of sigma mass. The first set is given by an arbitrary mass scale $\Lambda=16.48 \mathrm{MeV}$ which, together $\lambda_{1}^{2}=9.8554$ and $\nu_{1}=87.652 \mathrm{MeV}$ yields $m_{\pi}=138 \mathrm{MeV}$ and $m_{\sigma}=600 \mathrm{MeV}$. The second set is given by an arbitrary mass scale $\Lambda=16.48 \mathrm{MeV}$ which, together $\lambda_{1}^{2}=4.07$ and $\nu_{1}=79.447 \mathrm{MeV}$ yields $m_{\pi}=138 \mathrm{MeV}$ and $m_{\sigma}=400 \mathrm{MeV}$ as well as the original sigma model [21].

In Fig. 1, the effect of external magnetic field on the effective potential is plotted as a function of sigma field at $m_{\pi}=0$. Here three values of magnetic field $(B)$ are taken. Chiral limit is satisfied at $B=0$ $\left(m_{\pi}=0\right)$ and also for this boundary the spontaneous chiral symmetry breaking is noted. By increasing magnetic field, it is seen that the curves shift to upper values and the symmetry breaking is clearly appeared in the present model. If we increase magnetic field up to $e B=0.26 \mathrm{GeV}^{2}$ two minima values are recorded for the potential as depicted in Fig. 1. Two minima of the potential for $e B=0.26 \mathrm{GeV}^{2}$ are larger from the one at zero magnetic field. This indicates that the generation fermion mass increases with strongly increasing magnetic field.

In Fig. 2, effective potential of higher-order mesonic interactions is plotted as a function of sigma field at $e B=0.128 \mathrm{GeV}^{2}$. There is a qualitative agreement between the higher- order sigma model and the original sigma model. The curve of the original model shifts to lower values by including higher-order mesonic interactions, leading to a change in minima values of potential which gives the small generation fermion mass in comparison with original sigma model. The effective potential has two minima values of sigma field in the present model and the original sigma model. Therefore, the magnetic catalysis satisfies in the present model and agreement with NJL model [23], Schwinger-Dyson equation[24,25], and the logarithmic sigma model [32]. Indeed, one finds such an enhancement for the lattice QCD results calculated with physical pion masses $[33,34]$ at low temperatures.

In Fig. 3, the effective potential is plotted as a function of sigma field where the explicit symmetry breaking is included $\left(m_{\pi}=0.14 \mathrm{GeV}\right)$ at $e B=0.128 \mathrm{GeV}^{2}$. The explicit symmetry breaking is clearly appeared in the higher-order sigma model in comparison with the original sigma model. In addition, the two minima values of higher-order sigma model take smaller values than those of the original sigma model. 


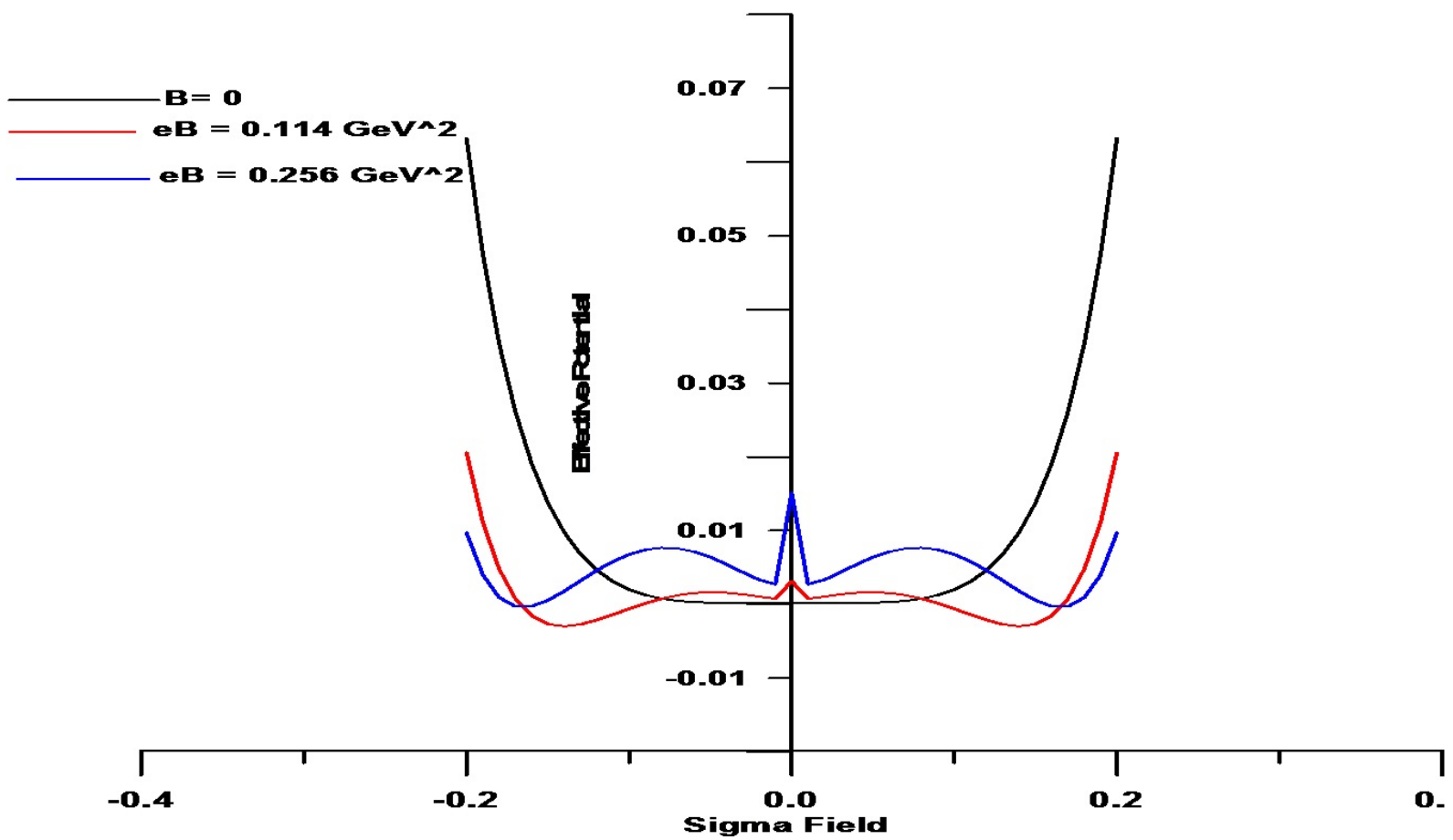

Figure 1. The variation of the effective mesonic potential as a function of sigma field for different magnetic field values $(\mathrm{B})$.

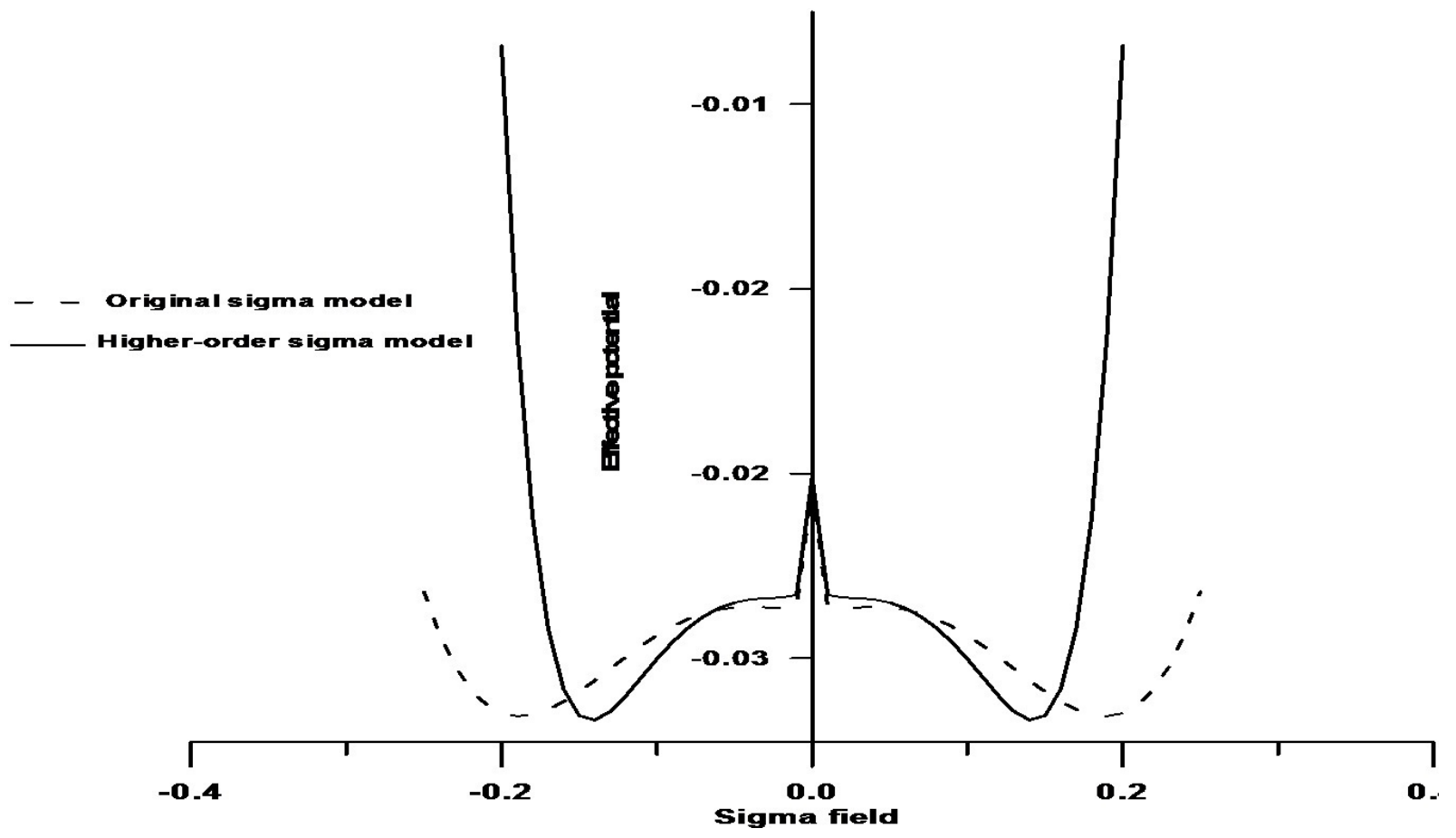

Figure 2. The variation of the effective mesonic potential as a function of sigma field for the higher-order of sigma model and original sigma model at $\mathrm{eB}=0.128 \mathrm{GeV}^{2}$ and $\mathrm{m}_{\pi}=0$.

The reason can be explained by the fact that the fermionic mass, generated from explicit symmetry breaking, is reduced in comparison with the original sigma model. Figs. $(2,3)$ represent the chiral limit 


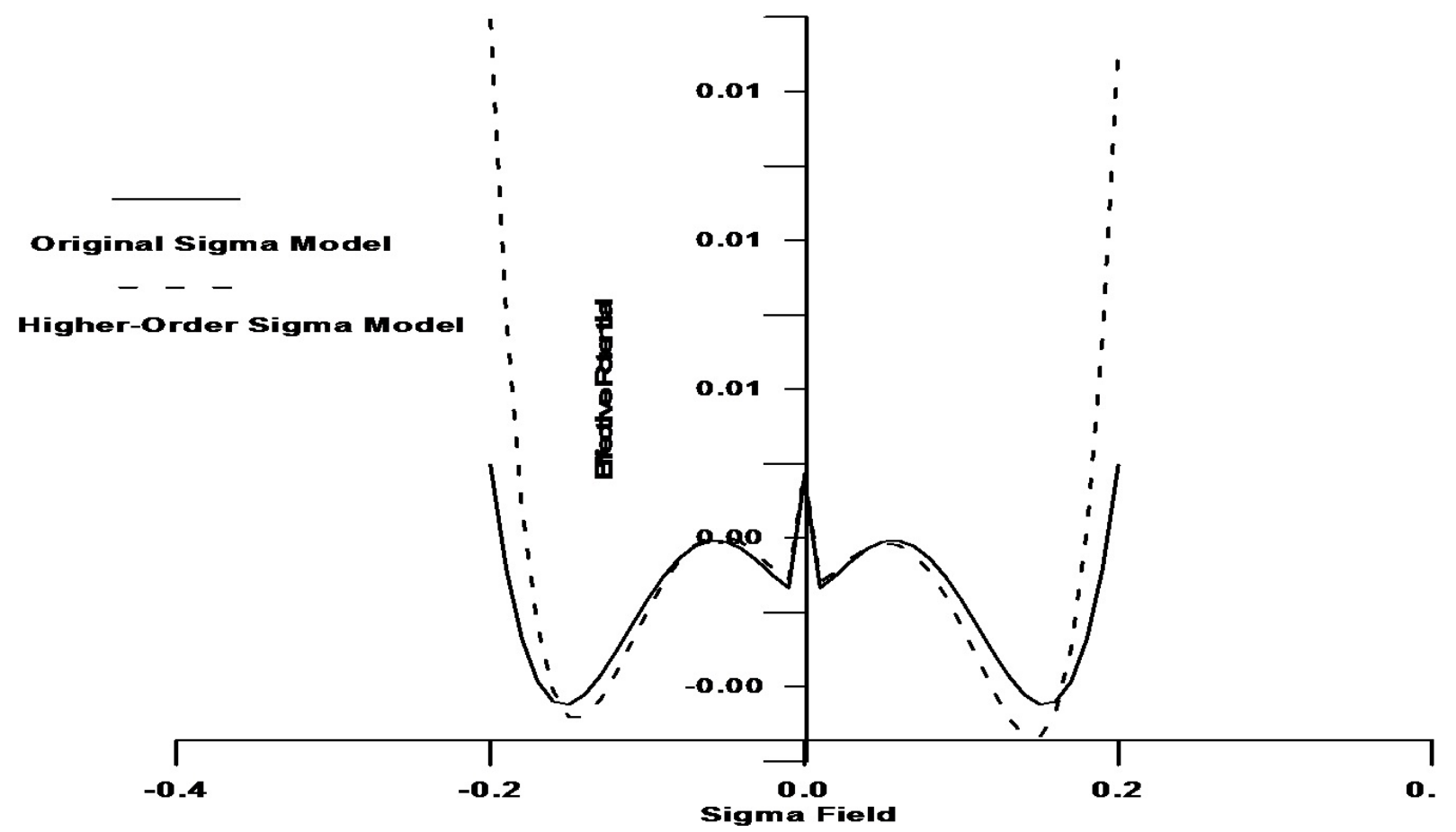

Figure 3. The variation of the effective mesonic potential as a function of sigma field for the present model and original sigma model at $\mathrm{eB}=0.128 \mathrm{GeV}^{2}$ and $\mathrm{m}_{\pi}=0.14 \mathrm{GeV}$

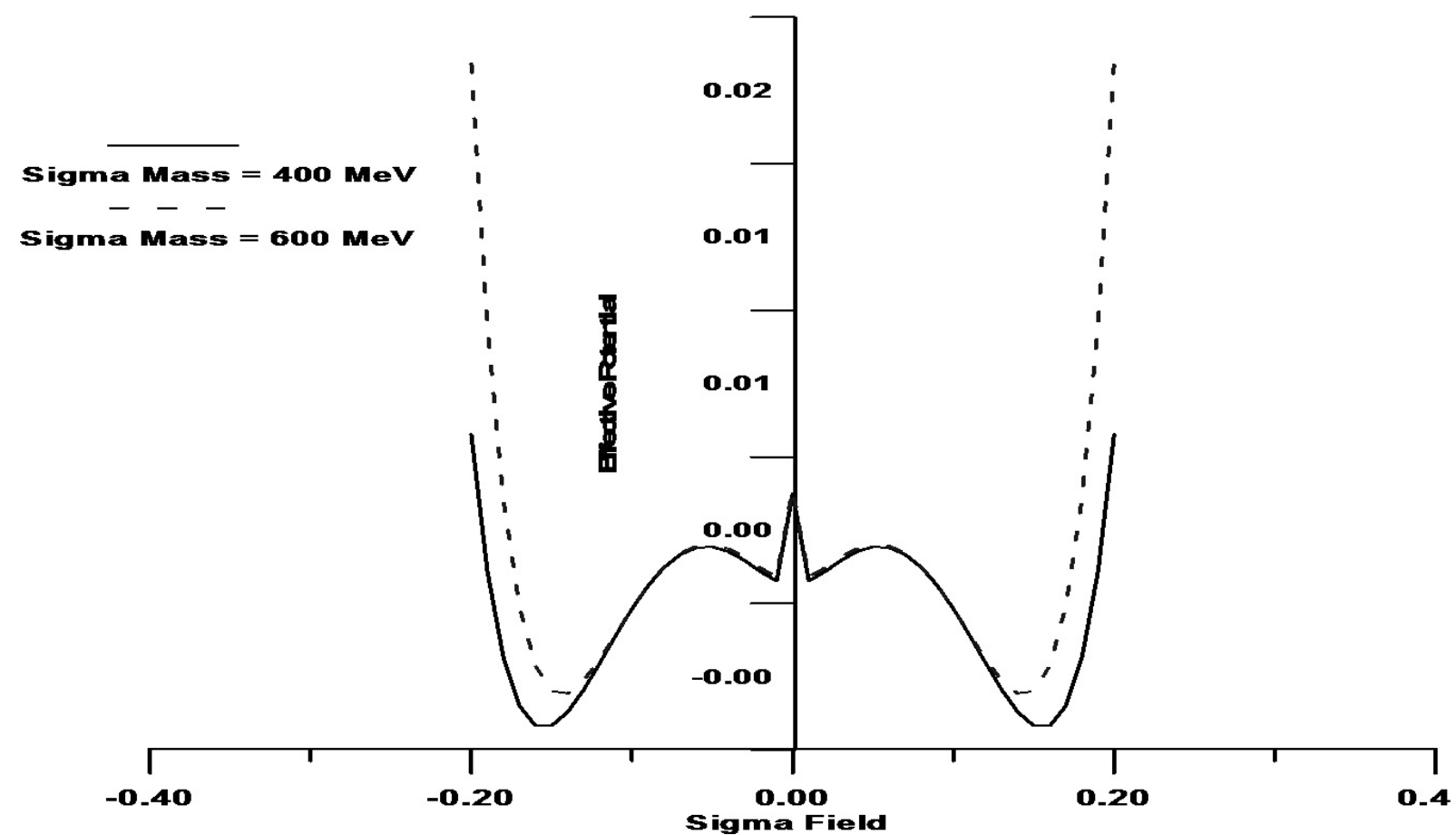

Figure 4. The variation of the effective mesonic potential as a function of sigma field for different sigma mass values at $\mathrm{eB}=0.128 \mathrm{GeV}^{2}$

and explicit symmetry breaking cases, respectively and indicate that the generated fermionic mass in comparison with original sigma model are reduced by the higher-order mesonic interactions. 
It is important to investigate the effect of the role of the free parameters on the phase transition and symmetry breaking. Fig. 4 shows the variation of the effective potential as a function of sigma field for two sigma mass values. It is seen that the curve shifts to lower values by decreasing sigma mass and the minima values of the potential are larger in comparison with their values at $m_{\sigma}=600 \mathrm{MeV}$. Thus, the generated mass increases by reducing sigma mass. The effect of coupling constant $(\mathrm{g})$ on the effective potential is drawn in Fig. 5. The curve shifts to lower values as the coupling constant $(g)$ gets a larger value with smaller two minima values of potential in comparison with their values at $g=4.5$. Therefore, the generated mass is small change by increasing coupling constant $g$ in the present of strong magnetic field.

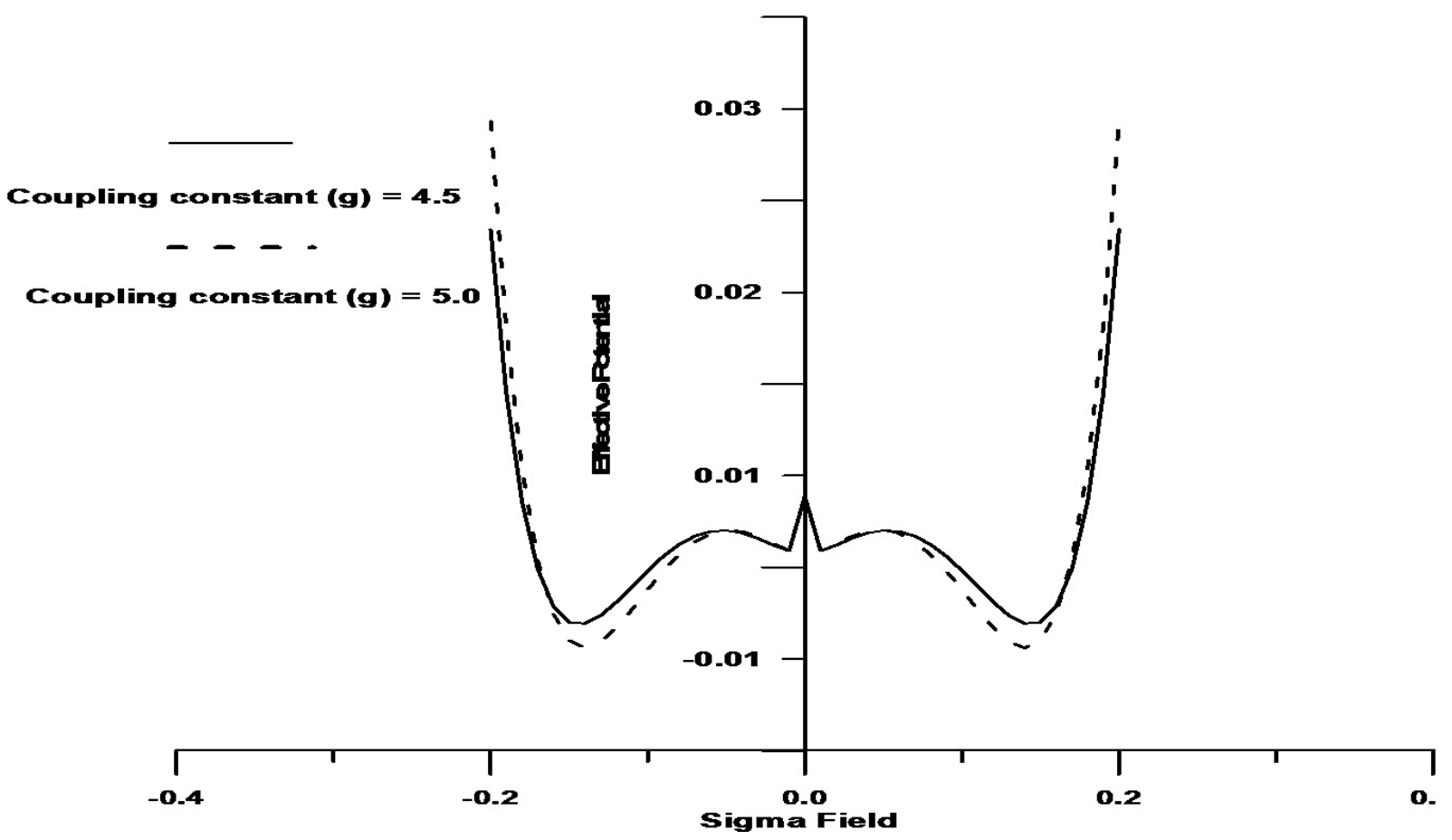

Figure 5. The effective mesonic potential is plotted as a function of sigma field for different values of coupling constant $\mathrm{g}$ at $\mathrm{eB}=0.128 \mathrm{GeV}^{2}$ and $\mathrm{m}_{\pi}=0$.

\section{Summary and conclusion}

We have investigated the higher-order mesonic interactions in the original sigma model on the chiral symmetry breaking in the presence of magnetic field. The novelty of this work is that the effect of the magnetic field is investigated in the framework of higher-order sigma model. In earlier works [11,30], this model was applied only to calculate the hadron properties at low energy without considering magnetic field. The obtained results show that the higher-order mesonic interactions reduce the generated baryonic mass, produced by chiral symmetry breaking as compared with the original sigma model. The effects of free parameters of sigma mass $\left(m_{\sigma}\right)$ and coupling constant $(g)$ play important role in magnetic catalysis in the present model. A comparison with other models is presented and shows that the obtained results are in a good agreement with other models and lattice QCD.

Therefore, the chiral higher-order sigma model enhances the magnetic catalysis at zero temperature. The generated quark mass is smaller in comparison with the original sigma model. The present study shows that the parameters of the model play a crucial role in the magnetic catalysis. We hope to extend the present model to finite temperature and chemical potential having a significant role to study the properties of the universe and neutron stars. 


\section{References}

1. V. S. Timoteo and C. L. Lima, Phys. Lett. B, vol. 635, p. 168, 2006.

2. M. Gell-Mann and M. Levy, Nuovo Cinmento, vol. 16, p. 705, 1960.

3. M. Birse and M. Banerjee, Phys. Rev. D, vol. 31, p. 118, 1985.

4. B. Golli and M. Rosina, Phys. Lett. B, vol. 165, p. 347, 1985.

5. K. Goeke, M. Harvey, F. Grummer, and J. N. Urbano, Phys. Rev. D, vol. 37, p. 754, 1988.

6. T. S. T. Aly, J. A. McNeil, and S. Pruess, Phys. Rev. D, vol. 60, p. 1114022, 1999.

7. W. Broniowski and B. Golli, Nucl. Phys. A, vol. 714, p. 575, 2003.

8. M. Abu-Shady, Int. J. Mod. Phys. A, vol. 26, p. 235, 2011.

9. —, Int. J. Theor. Phys., vol. 48, p. 1110, 2009.

10. —, Phys. Atom. Nuclei, vol. 73, p. 944, 2009.

11. - Int. J. Theor. Phys., vol. 48, p. 115, 2009.

12. N. Bilic and Nikolic, Eur. Phys. J. C, vol. 6, p. 515, 1999.

13. O. Scanvenius, A. Moscsy, I. N. Mishustin, and D. H. Rischke, Phys. Rev.C, vol. 64, p. 045202, 2001.

14. H. Mao, T.-Z. Wei, and J.-S. Jin, Phys. Rev. C, vol. 88, p. 035201, 2013.

15. M. Abu-Shady, Int. J. Mod. Phys. E, vol. 21, p. 1250061, 2012.

16. — Int. J. Theor. Phys., vol. 49, p. 2425, 2010.

17. — Int. J. Theor. Phys., vol. 50, p. 1372, 2011.

18. D. Kharzeev, K. Landsteiner, A. Schmitt, and H. U. Yee, Strongly interacting matter in magnetic fields. Springer, 2013.

19. B. S. Kandemir and A. Mogulkoc, Phys. Lett. A, vol. 379, p. 2120, 2015.

20. K. Kamikado and T. Kanazawa, JHEP, vol. 1, p. 129, 2015.

21. G. N. Ferrari, A. F. Garcia, and M. B. Pinto, Phys. Rev. D, vol. 86, p. 096005, 2012.

22. A. Goyal and M. Dahiya, Phys. Rev. D, vol. 62, p. 025022, 2011.

23. S. P. Klevansky and R. H. Lemmar, Phys. Rev. D, vol. 39, p. 3478, 1989.

24. I. A. Shushpanov and A. V. Smilga, Phys. Lett. B, vol. 16, p. 402, 1997.

25. — Phys. Lett. B, vol. 16, p. 351, 1997.

26. H. Suganuma and T. Tastsumi, Annals. Phys, vol. 208, p. 470, 1991.

27. K. G. Klimenko and T. Mat, Fiz., vol. 89, p. 211, 1991.

28. V. P. Gusynin, V. A. Miransky, and I. A. Shovkovy, Phy. Rev. Lett., vol. 73, p. 3499, 1994.

29. A. Feijoo, V. K. Magas, A. Ramos, and E. Oset, Phys. Rev. D, vol. 92, p. 076015, 2015.

30. M. Abu-Shady and M. Soleiman, Phys. Part. and Nucl. Lett., vol. 10, p. 683, 2013.

31. E. Elizalde, J. Phys. Math. Gen., vol. 18, p. 1637, 2013.

32. M. Abu-Shady, Appl. Math. Inf. Sci. Lett., vol. 4, p. 5, 2016.

33. G. S. Bali, F. Bruckmann, G. Endrodi, Z. Fodor, S. D. Katz, S. Krieg, A. Schafer, and K. K. Sz-abo, JHEP, vol. 1202, p. 044, 2012.

34. G. S. Bali, F. Bruckmann, G. Endrodi, Z. Fodor, S. D. Katz, and A. Schafer, Phys. Rev. D, vol. 86, p. 071502, 2012. 